\title{
Effect of oil-seed pressing residue on bread colour and texture
}

\author{
J. Tarek-Tilistyák \\ e-mail: judit.tilistyak@gmail.com
}

\author{
M. Tarek \\ e-mail: tarekshalaby2@gmail.com
}

\author{
College of Nyíregyháza, \\ Agricultural and Molecular Research and Service Institute, \\ H-4400 Nyíregyháza, Sóstói str. 31/B. Hungary \\ M. Juhász-Román \\ e-mail: rm@index.hu \\ Corvinus University of Budapest, \\ Department of Microbiology and Biotechnology, \\ H-1118 Budapest, Somlói str. 14-16. Hungary \\ J. Jekö \\ e-mail: jjozsi@nyf.hu \\ College of Nyíregyháza, \\ Agricultural and Molecular Research and Service Institute, \\ H-4400 Nyíregyháza, Sóstói str. 31/B. Hungary
}

\begin{abstract}
Cold-pressing residue of walnut kernel (WKR) and brown linseed (BLR) was applied in wheat flour blends at 100:0, 95:5 and 90:10 ratios, of which enriched breads were baked, then stored for 3 days at ambient temperature. Colour parameters and firmness of bread crumb were measured daily. Bavarian rye-bread (BR) and wholemeal multigrain bread (WMMG) were used as competitive, marketable breads for comparing tests.
\end{abstract}

Keywords and phrases: bread, walnut, linseed, pressing residue, by-product, colour, hardness. 
The studied oil-seed pressing residues (OSRs) resulted brown colour with different characteristics, depending both on the type of OSR and in comparison with marketed breads, too. The type and the ratio of OSR applied had no influence on the varying of crumb texture $(\mathrm{P}=0.107)$. WKR and BLR enrichment provided stable texture for breads with a 3-day shelf-life, independently from their addition ratio. BLR resulted in softer crumb than WKR; however, this difference was considered to be negligible $(\mathrm{P}=0.128)$. The WKR- and BLR-enriched breads stayed significantly softer at the end of storage time than the marketed breads $(\mathrm{P}=0.000)$. Our results indicate that competitive bakery goods can be produced using oil-seed pressing residue/wheat flour blends.

\section{Introduction}

Oil-seed pressing residues (OSRs) as by-products of vegetable oil industry are obtained in lower quantity typically in the technologies of SMEs (small and middle enterprises). In addition, OSRs are valuable, macronutrient-rich raw materials (Mueller et al., 2010; Vanhanen \& Savage, 2006); so, they could be a cheap and novel type of food components. Although the OSRs have found wide application in feed rations, it has already been demonstrated that these materials can be safe even for food application. From the manufacturing point of view, OSRs can have greater potential in bakeries because bread fortification is increasing in world-wide research (Preedy et al., 2011). Due to the high oil and dietary fibre content of OSRs, improved nutrients, colour, texture and the sensory profile of OSR-fortified breads are expected.

Marketability of foods like bread is influenced by consumer acceptation, basically by the texture of bread crumb. Besides, colourful baked products are rare. Different hues of white and brown colours are normally or traditionally accepted. Consumer demand toward multicoloured baked products is increasing regardless of artificial colouring additives. Black, blue, purple wheat grains are produced in small amounts for making specialty food (AbdelAl et al., 2006). Li et al. (2007) reported purple-wheat-bran-fortified muffin and demonstrated that such kind of bakery products have higher antioxidant capacity than whole-meal bread. In the 1990s, chemically extracted oil-seed meals were used and showed some colouring effect in breads, as Mansour et al. (1999) demonstrated the firming of pumpkin seed meal added breads. Nowadays, the application of oil-seed pressing residues in baked products, as recycled wastes, has novelty. The development of eco-innovative products is financed in the EU in the frame of Horizon 2020 strategy. 
Our research has been focused on developing nutritious, acceptable browntype bread products using pressing residue of walnut kernel and brown linseed. This objective was addressed by a comparative study of brown-type breads including similar breads commercially available with a 3-day shelf-life, using instrumental determination of crumb texture and colour. We have shown that the application of WKR and BLR can have great potential in the field of bakery innovation.

\section{Materials and method}

\section{$2.1 \quad$ Materials}

Cold-pressing residues of walnut kernel (moisture: $3.6 \%$, protein: $39.4 \%$, fat: 15.5\% ash: 4.9\%) were purchased from Tarpai Manufaktúra Kft. (Tarpa). Cold-pressing residues of brown linseed (moisture: $5.8 \%$, protein $29.9 \%$, fat: 18.9\%, ash: 7.1) were purchased from Solio Kft. (Szekszárd). The pressing residue was milled in laboratory, then passed through a sieve with apertures of $500 \mu \mathrm{m}$. Wheat flour (type: $0.8 \mathrm{~m} / \mathrm{m} \%$ ash) was acquired from the local market. Bavarian rye-bread and whole-meal multi-grain bread were purchased from the local market and were used for comparative study.

\subsection{Bread baking process and storage experiment}

Pressing residue was applied in wheat flour blends at 100:0, 95:5 and 90:10 ratios. To prepare dough, $1.5 \mathrm{~kg}$ flour blend, $2.0 \%$ salt, $1.5 \%$ active yeast and, depending on the type of OSR, variable quantities of water, necessary to comply with dough consistency, were mixed. The kneading was followed by leavening for 25 minutes at $28^{\circ} \mathrm{C}$. The dough was punched down; individual pieces of $580 \mathrm{~g}$ dough were cut and fermented in dough basket for 45 minutes at $30^{\circ} \mathrm{C}$ and $85 \%$ relative humidity. Round loaves were loosely baked at $230{ }^{\circ} \mathrm{C}$ for 35 minutes. The breads were left for cooling, then packed into plastic bags individually and stored at ambient temperature for 3 days.

\subsection{Instrumental determination of the physical properties of bread crumb}

Firmness and colour were measured daily and within 24 hours after baking for initial data. Bread slices with $1.25 \mathrm{~cm}$ width were cut right before measurement with fixed electric bread slicer. Firmness was determined with LFRA 
CT3 texture analyser (Brookfield, USA) according to AACCI 74-10.02 Compression Test. The colour was determined on a 5 -cm-diameter piece of bread crumb in Lab and LCh system with Colorite sph860 (CL150 model Z) spectrophotometer (Colorlite Gmbh, Germany) applying D65 illuminant and $10^{\circ}$ observer.

\subsection{Statistical evaluation of results}

Data were analysed by standard analysis of variance analysis. Tukey's HSD procedure was used for testing significant differences between means at the probability level $\mathrm{p}<0.05$. Harmonic mean sample size was 5.14 for texture and 3.79 for colour statistical analysis.

\section{Results and discussion}

\subsection{Effect of OSR addition on crumb colour and texture}

The OSR addition resulted complex changes in crumb colour. The results of colorimetric measurement can be seen in Table 1. The lightness parameter varied by the type of OSR and negatively correlated with the ratio of enrichment. The BLR addition positively correlated with hue and the redness value, while negatively with the yellowness. The WKR addition positively correlated with the redness and yellowness values, while the hue value did not change. Regarding total colour difference ( $\Delta \mathrm{E}$ values), the crumb colour of any studied OSR-enriched bread was a significantly different type of brown colour $(\mathrm{P}=0.000)$. OSR enrichment resulted in similarly dark breads with different hue and chroma from the studied marketed breads. However, WKR5 bread and BR bread are considered to be equivalent by total colour difference.

WKR- and BLR-enriched breads had soft crumb; the values varied between $2.27 \pm 0.2 \mathrm{~N}$ and $3.11 \pm 0.3 \mathrm{~N}$. Besides, the type and the ratio of the applied OSR had no influence on the varying of crumb texture $(\mathrm{P}=0.107)$. The crumb of BR bread was significantly firmer $(6.15 \pm 0.3 \mathrm{~N} ; \mathrm{P}=0.000)$ than the other ones. The initial firmness of WMMG bread and WKR-10, BLR-5 was $3.42 \pm 0.3 \mathrm{~N}$ and in the range of $2.54 \pm 0.2 \mathrm{~N}$, which was considered nearly identical $(\mathrm{P}=0.08)$. 


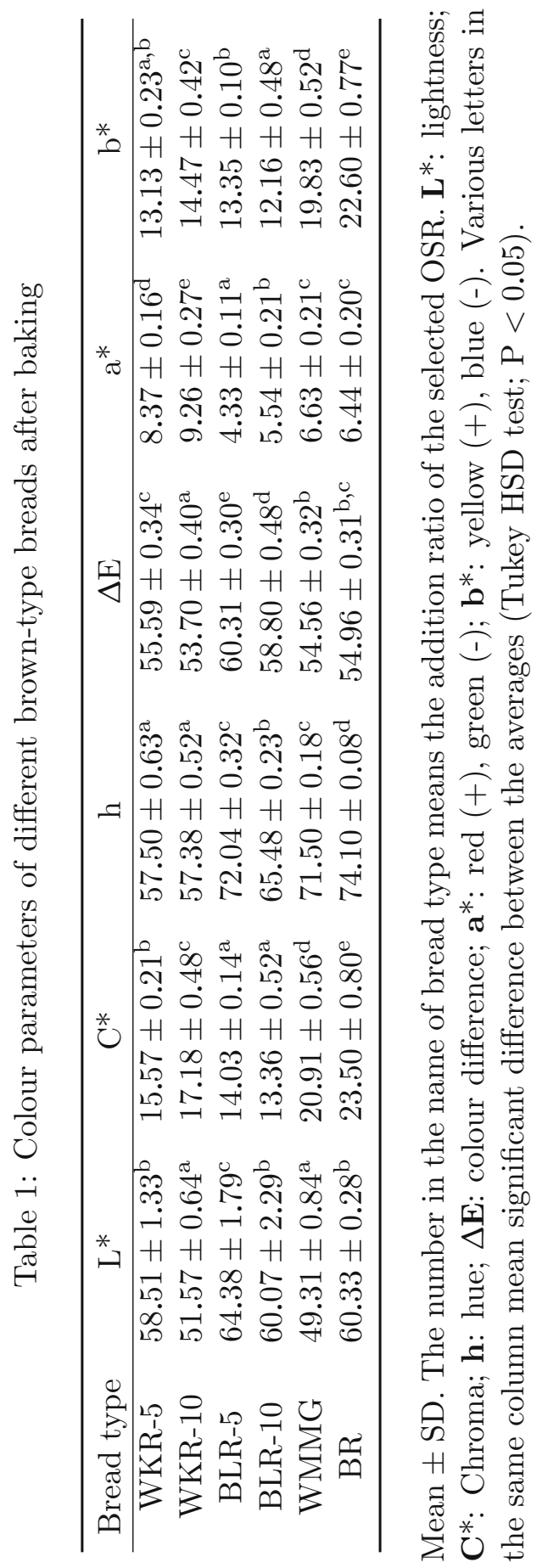




\subsection{Effect of storage on the quality of OSR-enriched breads}

During the storage term, either the colour or the firmness of OSR-enriched breads did not change significantly. WKR and BLR enrichment provided stable texture for breads with a 3-day shelf-life, independently from their addition ratio (see Fig. 1).

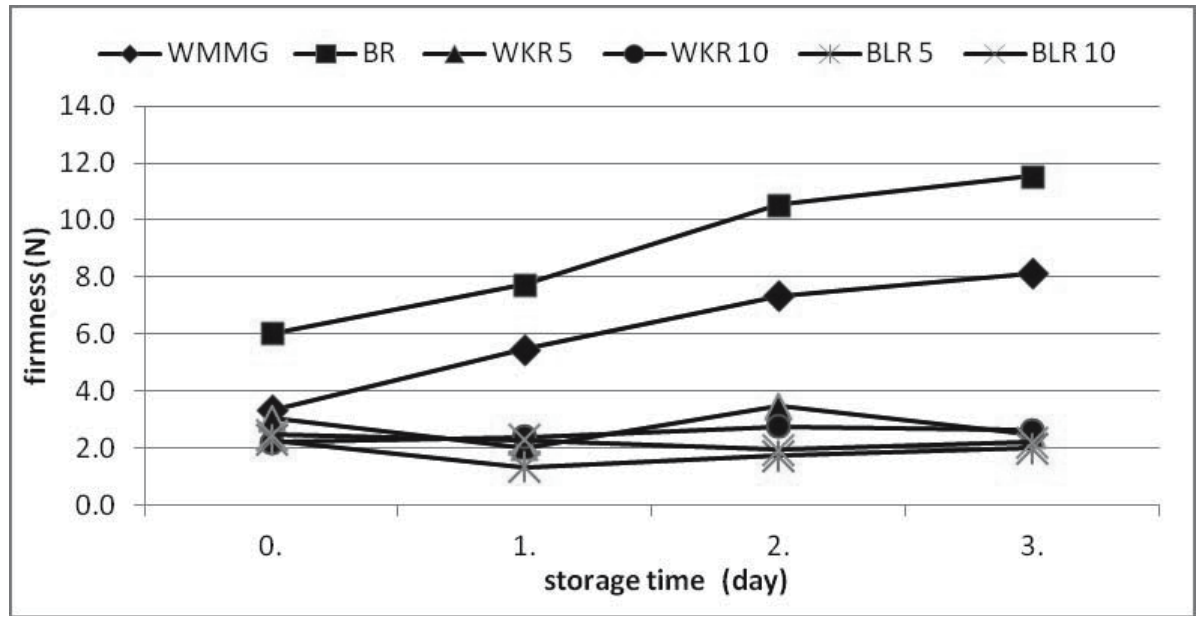

Figure 1: Bread crumb staling during a 3-day storage (average firmness in Newton)

The 3-day average firmness of different OSR-enriched breads was in the range of $1.8 \pm 0.3 \mathrm{~N}$ and $2.8 \pm 0.7 \mathrm{~N}$. BLR resulted in softer crumb than WKR; however, this difference considered to be negligible $(\mathrm{P}=0.128)$. Regarding the marketed breads, intensive staling of WMMG and BR breads was found; the 3-day average firmness was $6.1 \pm 0.5 \mathrm{~N}$ for WMMG bread and $9.0 \pm 0.5 \mathrm{~N}$ for BR bread.

The WKR- and BLR-enriched breads stayed significantly softer at the end of storage than the marketed breads $(\mathrm{P}=0.000)$. According to Ronda et al. (2014), enrichment of bread results in firmer crumb was stated and an increase in water retention during storage would be expected to decrease firmness. Linseed pressing residue, through its water-holding capacity (Mueller et al., 2010), can play the role of a stale-decreasing component in bread formulas.

We demonstrated in this experiment that the studied OSRs can be used as natural ingredients for double function as natural colouring and as texture 
improvers of enriched breads. Our result can be applied in the field of bakery product innovation and for expanding the assortment of breads.

\section{References}

[1] E.-S. M. Abdel-Aal, J. C. Young, I. Rabalski, Anthocyanin composition in black, blue, pink, purple and red cereal grains. J. Agric. Fd. Chem., 54. (2006) 4696-4704.

[2] W. Li, M. D. Pickard, T. Beta, Effect of thermal processing on antioxidant properties of purple wheat bran. Fd. Chem., 104. (2007) 1080-1086.

[3] K. Mueller, P. Eisner, Y. Yoshie-Stark, R. Nakada, E. Kirchhoff, Functional properties and chemical composition of fractionated brown and yellow linseed meal (Linum usitatissimum L.). J. Fd. Eng., 98. (2010) 453-460.

[4] E. H. Mansour, E. Dworschák, Zs. Pollhamer, Á. Gergely, J. Hóvári, Pumpkin and canola seed proteins and bread quality. Acta Alim., 28. (1999) 59-70.

[5] V. Preedy, R. Watson, V. Patel, Flour and breads and their fortification in health and disease prevention. Elsevier Inc. ISBN: 9780123808868. (2011).

[6] F. Ronda, J. Qulez, V. Pando, Y. H. Roos, Fermentation time and fiber effects on recrystallization of starch components and staling of bread from frozen part-baked bread. J. Fd. Eng., 151. (2014) 116-123.

[7] L. P. Vanhanen, G. P. Savage, The use of peroxide value as a measure of quality for walnut flour stored at five different temperatures using three different types of packaging. Fd. Chem., 99. (2006) 64-69. 